\section{COMMENT LA PEINTURE MULTIPLIE LE BLEU}

\section{Bernard Paquet}

RÉSUMÉ: Cet article analyse deux productions en peinture de type in situ réalisées selon un processus de multiple. Un modèle naît et génère ses propres variantes qui, en retour, étoffent son identité. II se conçoit alors dans le potentiel d'un registre de ressemblance. En ce sens, il est générique et entraîne la formation d'une série et un effet d'aura. Pratiqué hors des espaces traditionnels d'exposition, ce type de série peut entrainer par la force du multiple un changement de perception d'un lieu donné. L'émergence d'un modèle propre à la multiplication inscrit alors le fantasme du bleu dans le champ du multiple.

MOTS-CLÉS: peinture. multiple. série. modèle. aura. répétition.

La question du multiple est le plus souvent traitée sous l'angle des technologies de reproduction qui caractérisent, par exemple, la gravure et la photographie; une matrice physique ou numérique est conçue, formée, puis donne naissance à une série d'exemplaires qui sont identiques. Leur reproduction se fait nécessairement par contiguïté indicielle. Chaque copie doit avoir un contact avec la matrice, peu importe qu'elle soit une plaque d'impression ou un fichier jpeg originel. Or, en peinture, il est possible d'aborder le multiple sans matrice matérielle avec le but de réaliser une série potentiellement infinie. Celle-ci se compose alors de formes (ou d'œuvres) dont les ressemblances réciproques se fabriquent sur un mode plutôt iconique qui permet de jouer dans une zone de variations autour d'un modèle. Au contraire de la gravure, ce modèle naît avec l'instauration de l'œuvre et génère ses propres variantes qui, en retour, étoffent son identité. Ce même modèle se conçoit alors dans le potentiel d'un registre de ressemblance plutôt que comme une image précise. En ce sens, il est générique et entraîne la formation d'une série. Pratiqué hors des espaces traditionnels d'exposition, ce type de série peut entrainer par la force du multiple le changement de perception d'un lieu donné.

\section{MULTIPLE ET MODĖLE IN SITU}

C'est sous cet angle que j'analyse deux de mes productions récentes en peinture, réalisées lors de deux expositions de groupe in situ. Lors de ces installations temporaires de pièces peintes créées spécifiquement pour des lieux publics, j’ai exploité une stratégie du multiple, une première fois, dans le parc universitaire Valrose à Nice et, une seconde fois, à l'intérieur d'un édifice d'un des campus de l'Université de cette même ville.

La première installation, intitulée «Gouttes de mur» (figure I et 2), était composée de trente-six pièces fabriquées de peinture acrylique'. Chacune de ces pièces avait la forme d'une goutte, exécutée selon une composition répétitive dont la souplesse voulue permettait de varier le choix des couleurs et des proportions. Je peignais ces gouttes de bleus divers dans leurs parties hautes et, vers le bas, de teintes allant du violacé à l'ocre puis au vert. D'une pièce à l'autre, des changements se manifestaient dans les nuances de ces couleurs et dans les proportions entre la surface du bleu et celle des autres couleurs. L'effet visé était un sentiment général de bleu émergeant à grande échelle grâce à des nuances chromatiques. Mon intention était de suggérer le ciel et la terre à l'intérieur de chaque pièce puis de les suspendre par les trous d'évacuation de trois murs soulignant le relief du parc Valrose. J'entendais ainsi opposer la dureté grise du béton ou de la pierre à un matériau souple et coloré afin de rappeler le printemps, en accord avec le thème de la manifestation: Et si le printemps revenait? La logique fonctionnelle des orifices destinés à l'écoulement d'une eau de pluie salie se trouvait détournée au profil de l'idée d'un écoulement coloré étendu à une grande échelle. Je désirais que cet accrochage symbolise l'apparition et le jaillissement de la vie sur une matière endurcie et figée par l'hiver.

La seconde installation, intitulée «ll n'y a que le ciel qui nous tient» (figure 3), consistait à intervenir sur douze poutres reliant deux coursives à un très haut mur ${ }^{2}$ en les enrobant de toiles peintes à

\section{1}

"Gouttes de mur ", (36 peintures in situ), dans l'exposition de groupe Et si le printemps revenait?, organisée par no-made l'association et l'Université de Nice Sophia-Antipolis, campus de I'UFR Sciences, Parc Valrose, Nice, France, du 16 janvier au 20 mars 2010, (voir : www.no-made.eu).

\section{2}

«ll n'y a que le ciel qui nous tient»), (12 peintures in situ), dans l'exposition de groupe Si le printemps revenait II, organisée par no-made l'association et l'Université de Nice Sophia-Antipolis, campus Saint Jean d'Angély, Nice, France, du 9 mars au 29 avril 2011 (voir : www.no-made.eu). 
l'acrylique ${ }^{3}$. L'espace visé entre les murs du bâtiment administratif était gris et sombre, comme un couloir de la mort où on ne s'attarde guère. Je considérais que dans ce passage monochrome et terne, lever les yeux faisait espérer un peu de lumière du ciel mais, qu'au fil des jours, il fallait tout de même tenir, garder absolument le moral, espérer le retour du printemps ou de ce qu'il évoque : le ciel bleu, le soleil, les envolées chaudes et légères des oiseaux, les cerfs-volants et les ballons. Afin de donner une atmosphère de ciel de printemps à ce lieu, j’ai fabriqué des toiles qui présentaient six nuances de bleu et des motifs divers (nuages, oiseaux, ballons, etc.) suggérant la légèreté et le ciel. Une fois sur place, j’ai enrobé douze poutres de ces toiles en exploitant une stratégie du multiple qui visait à provoquer un sentiment général de bleu, nonobstant la présence de différents motifs figuratifs.

Ces deux œurres in situ ont bien en commun la multiplication de formes bleues dans des lieux minéraux et grisâtres. Bien que le processus de fabrication des divers éléments picturaux se soit déroulé en l'absence de matrice réelle, il suit néanmoins une démarche de répétition du même et de ses variations. Qu'en est-il de ce «même»? En réalité, il se manifeste comme une potentialité formelle et chromatique de développement qui laisse libre cours au plaisir de l'écart et de l'accumulation des variantes. Dans une telle démarche, c'est la multiplication des écarts qui finit paradoxalement par illustrer à posteriori la révélation d'un modèle à développer. L'exemple de la pratique du peintre Henri Cueco est à ce titre révélateur. À l'occasion d'une conférence qu'il donne à la Sorbonne en 19944, il expose les motifs de son travail sur une série de tableaux prenant pour modèle la pomme de terre, c'est-à-dire une idée générale de la représentation picturale de cet objet. Sa principale préoccupation était de peindre une suite de tableaux sous la gouverne d'un problème à résoudre qu'il résumait en ces termes : «comment ne pas peindre une pomme de terrell. Certes, ces propos semblent contradictoires mais ils ne le sont qu'en apparence. Ce que Cueco veut dire c'est plutôt comment ne pas peindre à la manière de ce qui a été fait jusqu'à maintenant et qui représente une pomme de terre ou encore comment peindre ce qui n'a jamais été encore vu comme l'image de ce qui sera idéalement nommé pomme de terre. Cette démarche entraine une capacité opératoire, en réalité quasi infinie, qui vise

3

L'auteur remercie le Conseil des arts et des lettres du Québec (CALQ) de son soutien financier pour la réalisation de ce projet.

À laquelle l'auteur avait assisté. non pas à cerner ce que serait le sens préexistant de ce légume mais à entamer une série ouverte à son avenir d'être. Celle-ci ne pourra, par définition, épuiser toutes les possibilités parce qu'elles croissent au fur et à mesure de l'instauration de nouvelles œuvres. Henri Cueco ne reproduit en rien un modèle; l'idée de la pomme de terre sera celle que la série fera naître.

Le modèle est toujours à construire, comme dans le cas des gouttes et des poutres bleues de mes installations. Car nos démarches respectives matérialisent par le multiple l'idée d'une forme colorée dont l'identité se précise au fur et à mesure de l'expansion de ses variations. II n'y a pas à l'origine de modèle à reproduire, mais au contraire, par la voie inverse, il y a production d'un modèle au travers d'une multiplication. A l'instar de la pomme de terre, les gouttes et les poutres bleues se tiennent à la fois par une proximité de ressemblance et par un écart de singularisation. Elles contribuent et continuent à amplifier la vision d'un modèle issu du multiple, à la manière de différents arbres dont l'ensemble procure une identité particulière à la forêt. D'une manière similaire, Francis Ponge ${ }^{5}$, considère qu'atteindre le cœur d'une chose consiste à utiliser les mots comme autant d'écrans qui, paradoxalement, révèlent par leurs sens respectifs, leur nombre et leur organisation ce cœur pourtant invisible et qui n'aurait aucune légitimité sans eux. II en est de même pour d'innombrables formes et motifs : paysage, nus féminins, portraits, ayant été peints au cours de l'histoire et qui ne nous autorisent pas pour autant à cerner de manière définitive ce qu'ils sont exactement, aujourd'hui, ni ce qu'ils pourraient être demain. Au travers des siècles, dans la peinture de ces motifs, quelque chose a été répété et multiplié, sans modèle originel, dans un registre de ressemblance qui n'est déterminé par aucune règle pré existante. Seule la masse du multiple induit ce registre de ressemblance qui soutient la répétition. Tout comme la forêt multiplie l'arbre qui ne relève pas d'une matrice mais bien d'une classe à la fois confirmée et enrichie par les singularités de chacun des individus qui l'intègrent.

Dès lors, la force du multiple relève non seulement du nombre de ses éléments mais encore de ce qui dépasse leur somme, selon la logique combinatoire d'une œurre associant des éléments distincts qui est $|+|=3$. Deleuze le met parfaitement en évidence lorsqu'il traite des deux termes solidaires que sont la différence et la répétition; il

5

Francis Ponge, Méthodes, Paris, Gallimard, 1961, pp. 207-207.

Gilles Deleuze, Différence et répétition (1968), Paris, PUF, $4^{\text {èm }}$ édition, 1981. 
y a là une résultante qui dépasse la simple accumulation d'une unité répétée. Au travers de sa propre succession, l'unité dessine sa propre différence pour se dissoudre dans l'ensemble de sa représentation. Deleuze va plus loin avec sa figure du rhizome lorsquill écrit que le multiple est traité comme un substantif lorsqu'il n'a plus de rapport avec l'Un'; il ne se résume pas à une qualification de l'unité. Ainsi, j'ai voulu que mes installations tendent vers cet état de substantif en établissant une régularité qui forme un plan de consistance. De telle sorte que l'ensemble de chaque oeurre amène une nouvelle connaissance visuelle qui oblitère inévitablement la reconnaissance de ses composantes.

\section{L'AURA DU MULTIPLE, SAMPLING DE BLEU}

Une telle émergence par le biais du multiple permet en outre de travailler à l'aura de l'œuvre au sens où Benjamin l'entend c'està-dire : «l'unique apparition d'un lointain, si proche soit-ilı. ${ }^{8}$. Par exemple, chaque goutte de mur renvoie, en tant que signe double, à l'idée d'un paysage et à celle d'une goutte. Chaque poutre peinte, renvoie à celle d'un volume horizontal et d'un ciel. Et cette idée demeure générale, de l'ordre de la catégorie plutôt que de la représentation précise d'un paysage donné, d'une goutte particulière ou d'un ciel particulier. Cependant, chaque pièce étant à la fois unique et représentative du groupe, le multiple entraîne une pléthore de versions qui élargissent d'autant la catégorie à laquelle nous sommes renvoyés. Mais au-delà de cette catégorie, les deux ensembles acquièrent également le statu de signe et, par conséquent, apparaissent comme la concrétisation d'un lointain (l'équivalent du cœur de la forêt) qui est cependant moins identifiable que celui de leurs composantes respectives. Ce lointain se situe dans le sillage du sentiment général de coulées bleues, voire d'un simple effet rythmique, émergeant lui-même de sentiments individuels de gouttes. Dans de telles circonstances, le multiple n'est plus une perte d'aura, comme Benjamin l'a entrevu pour l'unicité de l'œurre dans la tradition mais, au contraire, la confirmation de l'apparition de quelque chose d'unique. Car, comme le précise ce dernier dans un retournement capital, l'impact des moyens de reproduction caractérise $॥ \ldots$ une perception devenue assez apte à sentir tout

\section{7}

Gilles Deleuze, Rhizome, Paris, Minuit, 1976, p. 21.

8

Walter Benjamin, L’homme, le langage et la culture, Paris, Denoël/Gonthier, 1971, p.70. ce qui est identique dans le monde pour être capable de saisir aussi, par la reproduction, ce qui est uniquen'. Ainsi, selon moi, le seul fait de reproduire des variations de gouttes ou de poutres dans un lieu donné signifie qu'il y a là quelque chose d'unique qui émerge. La distance à laquelle se situe le spectateur est par conséquent déterminante.

De même, qu'en musique électronique, la répétition d'une phrase musicale de la tradition par le sampling permet à l'aura de réapparaître sous un jour nouveau car ule sample mis en boucle et répété à l'infini ne tue pas sa propre aura) ${ }^{10}$, la répétition opérée par le sampling serait paradoxalement ce qui permet de ressentir l'aura d'une musique.

Cette idée du sampling s'inscrit dans une optique de la pratique contemporaine où l'artiste peut être comparé à un programmeur, ou bien à un metteur en scène qui dispose des objets et images que le monde met à sa disposition, pour mieux les associer librement avec le but de faire oeuvre ${ }^{10}$. Ce dernier propose donc des contiguités, établit des liens, voit des continuités entre des objets, là où les autres ne voient que des points isolés et sans rapport aucun. Dans mon travail, l'équivalent du procédé de sampling consiste à joindre des éléments picturaux parce qu'ils se ressemblent, ce qui me permet de tabler sur les effets de la répétition. Mais ici ce n'est pas l'objet de la stricte reproduction technologique comme l'entend Benjamin qui est en jeu, mais bien le processus de reproduction au sens élargi d'un registre de ressemblance. En ce sens, la forme de la goutte et celle de la poutre sont des contenants à remplir de peinture. Elles fonctionnent comme de véritables gènes manipulés en tant que potentialités de production qui favorisent un processus de reproduction afin de donner du corps à une entreprise réalisée à grande échelle dont le but est d'habiter un lieu donné par un sentiment de bleu et un effet de surprise. Au moment de la production d'une goutte, je vois toutes celles que j’ai déjà réalisées et j'entrevois celles qui peuvent venir. Mais il ne s'agit pas d'une duplication au sens strict de la génétique car chaque goutte présente

9

Ibid., p. 71.

10

A. Laumonier, "Courtesy of....", in Art Press, Techno. Anatomie des cultures électroniques, Hors série no 19, 1998, p. 85.

11

N. Bourriaud, "La mutuelle des formes», in Art Press, Techno. Anatomie des cultures électroniques, Hors série no 19, 1998, pp. 163-169. 
sa singularité et laisse entrevoir la potentialité du multiple à venir selon un mode qui se compare à celui d'une mise en abyme. En réalité, chaque goutte contient en puissance toutes les possibilités de différence des autres gouttes; ce qui est aussi vrai pour les poutres.

\section{ÉRIALITÉ BLEUTÉE}

La succession des gouttes et des poutres de mes deux installations obéit également à la stratégie artistique de la sérialité que le modernisme a largement mis en exergue et que les avant-gardes ont exploitée $e^{12}$. Jeu intentionnel dans lequel le hasard a très peu de place, la pratique de la série obéit ici à un certain code (forme de goutte, enrobage de poutres par une toile, disposition régulière, couleur bleue) qui est dirigé par une volonté de cohésion. Le retour assumé du code assure l'homéostasie de chacune des deux oeurres. À l'intérieur de l'ensemble de l'installation des gouttes, il n'y a pas de hiérarchie car le processus suit un système II...essentiellement distributionnel, dans le temps perceptif et dans l'espace plastique»/13 qui est rythmé à l'origine par la régularité des trous existant sur les trois murs du parc Valrose. Toute permutation y est permise par le registre de ressemblance alors que chaque goutte, pourtant unique, peut néanmoins être substituée par une autre sans véritable conséquence. Tout élément de ce multiple structuré efface ses propres caractéristiques devant sa manipulation ${ }^{14}$. Dès lors, «Gouttes de murn répond à une définition de l'art sériel de l'avant-garde, qui est : «fragmentation élémentaire où se dissolvent les identités; commutabilité des éléments; règles combinatoiress $)^{15}$.

II en est de même pour l'installation des poutres dans II n'y a que le ciel qui nous tient, avec la différence qu'elle comporte une certaine hiérarchie qui suit les six nuances de bleus qui s'entrecroisent sur les deux niveaux mais qui ne permet pas le jeu des permutations de la première œurre.

12

Marc Le Bot, Figures de l'art contemporain, Paris, UDF, coll. 10/18, 1971.

13

Ibid., p. 284.

14

Ibid. ,p. 285.

15

Loc. cit.
Le multiple pratiqué dans cet esprit de la série vise, à posteriori, un ordre idéal, celui du bleu, contrastant avec l'ordre établi du gris des lieux. C'est selon Marc Le Bot, la vérité de l'art qui s'accompagne de cet ordre en tant que résultat, transcendant au domaine concret des éléments qui composent le multiple ${ }^{16}$. Pour lui, la logique combinatoire de la sérialité de l'art contemporain se définit par l'absence de modèle ${ }^{17}$, ce qui n'empêche nullement le processus de jouer sur des articulations de différences selon un mode naissant. C'est là le sens des propos de Cueco : il n'y aucun modèle qui précède l'instauration de l'œuvre et qui serait à reproduire, en tant que référent externe. Bien au contraire, dans l'esprit postmoderne, l'œuvre se construit elle-même vers un modèle et invente ses propres règles : une forme est mise au point et induit la multiplication de ses variations. Une œuvre apparaît dès lors comme son propre modèle.

Dans mes installations, lidée originelle de susciter un fort sentiment de bleu a entrainé la nécessité de créer des formes plastiques: gouttes et rectangles pour les poutres. Les lieux visés, extérieur et intérieur, exigeaient des installations à grande échelle selon leurs caractéristiques : murs scandés de trous et poutres se succédant avec régularité. Cet impératif s'accompagnait d'une logique de sérialité supposant un système axé sur la multiplication du bleu, en fonction d'une certaine hiérarchie et de possibilités combinatoires. Comme le souligne Paul Klee ${ }^{18}$, utoute mise en oeurre est relation du particulier au généralı, sa cohésion se constitue en vertu des rapports liant les parties entre elles et à l'ensemble. Dans cette optique, l'idée initiale des ces deux installations reposait sur un élément formel et chromatique facilement reproductible par la largesse de ses variations. J'ai conçu l'élément de départ comme le début d'une somme, soit 36 pour les murs et 12 pour les poutres, tout en tablant sur la possibilité que cet élément crée une série et que cette série soit le départ d'une oeurre.

J'ai ainsi envisagé une dimension supplémentaire qui ne pouvait être prévue par la simple régularité de l'organisation sérielle dans la mesure où des éléments devaient s'articuler dans un mouvement global de formation. Installer une œuvre en fonction d'un lieu avait donc pour but de créer un modèle.

16

Ibid., p. 287

17

Ibid., p. 290.

18

Paul Klee, Théorie de l'art moderne, Paris, Denoël/Gonthier, 1973, p. 60. 
Le tout était de donner une forme à la multiplication pour inscrire le fantasme du bleu dans le champ du multiple.

\section{RÉFERÊNCES}

BENJAMIN, W. L'homme, le langage, la culture. Paris: Denoë//Gonthier, 1971.

BoURRIAUD, N. «La mutuelle des formes» in Art Press, Techno. Anatomie des cultures électroniques, Hors série $n^{\circ} 19,1998$,

DELEUZE, G. Différence et répétition (1968). Paris : PUF, $4^{\text {ème }}$ éd. ,198I.

DELEUZE, G. Rhizome. Paris : Minuit, 1976.

KLEE, P. Théorie de l'art moderne. Paris : Denoël/Gonthier, 1973.

LAUMONIER, A. "Courtesy of....), in Art Press, Techno. Anatomie des cultures électroniques, Hors série $n^{\circ} 19,1998$.

LE BOT, M. Figures de l'art contemporain. Paris : UDF, coll. 10/18, 1977.

PONGE, F. Méthodes. Paris : Gallimard, 1961.

LÉGENDES DES ILLUSTRATIONS

Les images de cet article son placées dans sa version porugaise.

Figure I: Bernard Paquet, Gouttes de mur, 2010, peinture acrylique sur médium acrylique, 36 pièces de $110 \times 50 \mathrm{~cm}$ chacune, pour l'exposition Et si le printemps revenait?, Parc Valrose, Nice, France.

Figure 2: Bernard Paquet, Gouttes de mur, 2010, peinture acrylique sur médium acrylique, 36 pièces de $110 \times 50 \mathrm{~cm}$ chacune, pour l'exposition Et si le printemps revenait?, Parc Valrose, Nice, France.

Figure 3: Bernard Paquet, II n'y a que le ciel qui nous tient, 201I, 12 peintures acrylique sur toile, $164 \times 140 \mathrm{~cm}$ chacune, pour l'exposition Et si le printemps revenait II, Campus Saint Jean d'Angély de l'université de Nice Sophia Antipolis, Nice, France.

BERNARD PAQUET: Professeur titulaire à l'École des arts visuels de l'Université Laval à Québec, Canada, où il enseigne la peinture. Docteur en Arts et Sciences de l'Art (Paris I Panthéon-Sorbonne), DSAP de l'École Nationale Supérieure des beaux-arts de Paris. Une trentaine d'expositions solo et de groupe (Canada, Brésil, France, Monaco, Tunisie). Des conférences et publications dans des revues, livres et catalogues (Brésil, Canada, France, Martinique, Royaume-Uni, Suisse, Tunisie). Sa pratique porte sur le principe de couches en peinture, la peinture d'installation, le visage humain et le métissage du dessin et de la photographie. 
